\title{
Conformal Changes and Geodesic Completeness
}

\author{
John K. Beem \\ Department of Mathematics, University of Missouri, Columbia, Missouri 65201, USA
}

\begin{abstract}
Let $(M, g)$ be a causal spacetime. Condition $N$ will be satisfied if for each compact subset $K$ of $M$ there is no future inextendible nonspacelike curve which is totally future imprisoned in $K$. If $M$ satisfies condition $N$, then whenever $E$ is an open and relatively compact subset of $M$ the spacetime $E$ with the metric $g$ restricted to $E$ is stably causal. Furthermore, there is a conformal factor $\Omega$ such that $\left(M, \Omega^{2} g\right)$ is both null and timelike geodesically complete. If $M$ is an open subset of two dimensional Minkowskian space, then $M$ is conformal to a geodesically complete spacetime.
\end{abstract}

\section{Introduction}

Causality is playing an important role in the study of relativity. It is useful in the investigation of black holes $[1,2]$ and in cosmology $[3,4]$.

A spacetime which remains causal under slight perturbations of the metric is called stably causal. This is one of the most reasonable causality conditions since quantum effects imply that measurements are always imprecise.

It is an interesting fact that many spacetimes which are not themselves stably causal have relatively large subsets which are stably causal. The question arises of how to decide if a given subset of a causal spacetime is stably causal. The first interesting result of this paper is that if $(M, g)$ satisfies the nonimprisonment condition $N$, then any open subset $E$ of $M$ with compact closure $\bar{E}$ is stably causal. This means that when condition $N$ is valid and $(M, g)$ is not stably causal the stability condition breaks down near the boundary of $M$. A corollary of our first theorem is that if $M$ satisfies condition $N$ and $\mu$ is a bounded measure on $M$, then whenever $\varepsilon>0$ is given there is a closed subset $F$ of $M$ such that $\mu(F)<\varepsilon$ and $M-F$ is stably causal.

Let $\Omega$ be a positive real valued function on $M$. The two metrics $g$ and $\Omega^{2} g$ on $M$ are said to be conformally equivalent. The function $\Omega$ is called a conformal factor. The introduction of a conformal factor does not change the causality of $M$ since a curve is timelike (null) for $g$ if and only if it is timelike (null) for $\Omega^{2} g$. On 
the other hand, the causality of $M$ determines the metric $g$ up to some conformal factor, [1]. The study of conformal geometry on $M$ is equivalent to the study of causality on $M$.

For positive definite Riemannian metrics one may always find a conformally equivalent metric which is geodesically complete, [5]. The corresponding statement is not true for all causal spacetimes. However, some results have been obtained using stronger assumptions. Seifert $[6,7]$ has shown that if $(M, g)$ is stably causal then $M$ is conformal to a spacetime which has all future directed nonspacelike geodesics complete. Clarke [8] has shown that a strongly causal spacetime may be made null geodesically complete. In the present paper we show that if $(M, g)$ satisfies condition $N$, then there is a conformal factor $\Omega$ such that every nonspacelike geodesic of $\left(M, \Omega^{2} g\right)$ is complete. Stable causality implies strong causality and strong causality implies condition $N$. Consequently, our result is an improvement on the previous results.

In the proof we use an expanding sequence of subsets of $M$ such that each set is open and relatively compact. Each member of the sequence is stably causal and hence has a cosmic time function. This cosmic time function allows us to construct a conformal factor $\Omega_{k}$ which is unity except for the $k^{\text {th }}$ member of the sequence. The final factor $\Omega$ will be the infinite product of the functions $\Omega_{k}$.

\section{Preliminaries}

Let $(M, g)$ be an $n$-dimensional spacetime. This means $M$ is a $C^{\infty}$ Hausdorff manifold with a countable base, a Lorentz metric $g$ of signature $(-,+, \ldots,+)$ and a time orientation. The time orientation will always be determined by a $C^{\infty}$ (future directed) timelike vector field $X$ on $M$. We do not require that $M$ be connected.

A causal spacetime is a spacetime which has no closed nonspacelike curve [1].

A future directed curve $x(t)$ for $a<t<b$ is said to have a future endpoint $p$ if for each neighborhood $U$ of $p$ there is some $t_{1}$ with $a \leqq t_{1}<b$ such that $x(t) \in U$ for all $t_{1} \leqq t<b$. A nonspacelike future directed curve is future inextendible if it has no future endpoint [1].

If $K$ is a subset of $M$ and $x(t)$ is a future directed curve, then $x(t)$ is totally future imprisoned in $K$ if there is some $t_{1}$ such that $x(t) \in K$ for all $t \geqq t_{1}$. Past imprisonment is defined in a similar fashion. An example in [1] due to Carter shows that some causal spacetimes contain compact subsets which have nonspacelike curves totally future imprisoned in them.

Condition $N$. The causal spacetime $(M, g)$ will satisfy condition $\mathrm{N}$ if for each compact subset $K$ of $M$ there is no future inextendible nonspacelike curve $x(t)$ which is totally future imprisoned in $K$.

In Section 3 it will be shown that the exclusion of total future imprisonment for all compact subsets is equivalent to the exclusion of total past imprisonment for all compact subsets. Notice that condition $\mathrm{N}$ does not exclude the possibility of partial future imprisonment.

The spacetime $(M, g)$ is strongly causal if for each $p \in M$ and each open neighborhood of $p$ there is a smaller neighborhood of $p$ such that no nonspacelike 
curve intersects the smaller neighborhood more than once. It is known [1] that a strongly causal spacetime satisfies condition $\mathrm{N}$.

The spacetime $(M, g)$ is stably causal if the metric $g$ has a neighborhood in the $C^{0}$ open topology such that there are no closed timelike curves in any metric belonging to the neighborhood. The $C^{0}$ open topology is defined in terms of $T S_{2}^{0}(M)$, the bundle of symmetric tensors of type $(0,2)$ over $M$. A Lorentz metric is a cross section $\hat{g}: M \rightarrow T S_{2}^{0}(M)$ of this bundle. If $V$ is an open subset of the bundle $T S_{2}^{0}(M)$, then $O(V)$ is defined to be all $C^{0}$ Lorentz metrics $\hat{g}$ such that $\hat{g}(M)$ is contained in $V$. Sets of the form $O(V)$ form a basis of the $C^{0}$ open topology. A spacetime $M$ is stably causal if and only if there is a function $f$ on $M$ whose gradient is everywhere timelike, see [1]. The function $f$ is called a cosmic time function. When cosmic time functions exist they are not unique. Stable causality implies strong causality.

Consider now a point $p$ lying in an open set $U$ of $M$. The causal future of $p$ in $U$ for the metric $g$ will be denoted by $J^{+}(p, U, g)$. This set consists of all points $q \in U$ such that there exists a future directed nonspacelike curve $x(t)$ in $U$ from $p$ to $q$.

\section{Stably Causal Subsets}

Let $X$ be a $C^{\infty}$ timelike future directed vector field on $M$. For each fixed $\lambda \geqq 0$ define the Lorentz metric $g[\lambda]$ on $M$ by

$$
g[\lambda](Y, Z)=g(Y, Z)-\lambda g(X, Y) g(X, Z)
$$

where $Y$ and $Z$ are tangent vectors of $M$. The vector field $X$ remains timelike for the metric $g[\lambda]$ and gives a time orientation to $(M, g[\lambda])$. If the vector $Y$ is orthogonal to $X$ for the metric $g=g[0]$, then $Y$ remains orthogonal to $X$ for the metric $g[\lambda]$. It follows that $g[\lambda]$ is nondegenerate for all $\lambda \geqq 0$. Furthermore, the light cone of $g[\lambda]$ decreases toward the light cone of $g$ as $\lambda$ decreases toward zero. If $\lambda_{1}>\lambda_{2}$ and $Z$ is a future directed nonspacelike vector for $g\left[\lambda_{2}\right]$, then $Z$ is a future directed timelike vector for $\lambda_{1}$.

In the next Lemma we show that locally the causality of $g[\lambda]$ converges to the causality of $g$ as $\lambda$ converges to zero.

Lemma 1. Let $\lambda_{m} \rightarrow 0^{+}$and $p \in M$. There is a neighborhood $U$ of $p$ such that if $r_{m} \rightarrow r \in U$ and $q_{m} \rightarrow q \in U$ with $q_{m} \in J^{+}\left(r_{m}, U, g\left[\lambda_{m}\right]\right)$, then $q \in J^{+}(r, U, g)$.

Proof. Let $\exp [\lambda]: T(M) \rightarrow M$ be the exponential map for $g[\lambda]$. Let $W$ be an open subset of the tangent bundle $T(M)$ with $\bar{W}$ compact, $p \in \exp [0](W)$ and assume $\exp [0]$ is defined on an open set containing $\bar{W}$. There is some $c>0$ with $\exp [\lambda]$ defined on an open set about $\bar{W}$ for all $0 \leqq \lambda<c$. Let $\left(u^{1}, \ldots, u^{n}\right)$ be normal coordinates about $p$ and $\left(u^{1}, \ldots, u^{n}, v^{1}, \ldots, v^{n}\right)=(u, v)$ corresponding coordinates on $T(M)$ using the natural basis. The map $\exp [\lambda](u, v)$ is a $C^{\infty}$ function of $u, v$ and $\lambda$ for all $(u, v) \in W$ and $0 \leqq \lambda<c$. For each fixed $\lambda$ there is a convex normal neighborhood of $p$ for $g[\lambda]$. Since $\exp [\lambda](u, v)$ is $C^{\infty}$ it is possible to find a $c_{1}>0$ and a set $U C \exp [0](W)$ such that $U$ is a convex normal neighborhood of $p$ for $g$ and such that for all $0 \leqq \lambda<c_{1}$ the set $U$ lies in a set $U[\lambda]$ which is a convex normal neighborhood for $g[\lambda]$. 
Let $x(t)=\exp [0]\left(u_{0}, t v_{0}\right)$ for $0 \leqq t \leqq 1$ be the unique geodesic for $g$ in $U$ with $x(0)=r$ and $x(1)=q$. For sufficiently large $m$ there exists $u_{m}$ and $v_{m}$ such that $\exp \left[\lambda_{m}\right]\left(u_{m}, t v_{m}\right)$ for $0 \leqq t \leqq 1$ is a geodesic of $g\left[\lambda_{m}\right]$ from $r_{m}$ to $q_{m}$. Furthermore, $u_{m} \rightarrow u_{0}$ and $v_{m} \rightarrow v_{0}$. Since $q_{m} \in J^{+}\left(r_{m}, U, g\left[\lambda_{m}\right]\right.$ and $U \subset U\left[\lambda_{m}\right]$, the tangent vector $\left(u_{m}, v_{m}\right)$ is future directed nonspacelike for $g\left[\lambda_{m}\right]$. It follows that $\left(u_{0}, v_{0}\right)$ is a future directed nonspacelike vector for $g$ and $q \in J^{+}(r, U, g)$. This establishes the Lemma.

When $(M, g)$ is a causal spacetime and $E$ is an open subset of $M$ we will use $(E, g)$ to denote the spacetime obtained by using the metric $g$ restricted to the manifold $E$.

Theorem 2. Let $(M, g)$ be a causal spacetime which satisfies condition $N$. If $E$ is an open subset of $M$ with compact closure $\bar{E}$, then $(E, g)$ is stably causal.

Proof. Assume $E$ is not stably causal and let $\lambda_{m}$ be a decreasing sequence of numbers with $\lambda_{m} \rightarrow 0$. For each $m$ there is a closed curve $\sigma_{m}(t)$ for $0 \leqq t \leqq 1$ lying in $E$ with $\sigma_{m}(0)=\sigma_{m}(1)$ and $\sigma_{m}$ timelike future directed for the metric $g\left[\lambda_{m}\right]$. Extend the parametrization of $\sigma_{m}$ by defining $\sigma_{m}(t+k)=\sigma_{m}(t)$ for all $0 \leqq t \leqq 1$ and integers $k$. The curves $\sigma_{m}$ are both future and past inextendible nonspacelike curves for $g\left[\lambda_{1}\right]$. For each $m$ choose a point $p_{m} \in \sigma_{\underline{m}}$. The sequence $\left\{p_{m}\right\}$ must have a subsequence converging to some $p \in \bar{E}$ since $\bar{E}$ is compact. Lemma 6.2.1 of [1] implies the curves $\sigma_{m}$ must have a limit curve $\sigma$ which contains $p$, is nonspacelike for $g\left[\lambda_{1}\right]$ and is both future and past inextendible in $M$. Since $\sigma_{m} \subset E$ we have $\sigma \subset \bar{E}$. Lemma 1 implies that $\sigma$ is nonspacelike for the metric $g$. The curve $\sigma$ is future and past inextendible for $g$ since it is future and past inextendible for $g\left[\lambda_{1}\right]$. The curve $\sigma$ contradicts condition $\mathrm{N}$ and establishes the Theorem.

Corollary 3. Let $(M, g)$ be a strongly causal spacetime. If $E$ is an open and relatively compact subset of $M$, then $E$ is stably causal.

Theorem 4. Let $(M, g)$ be a causal spacetime. The following three conditions are equivalent.

1. Condition $N$.

2. For each compact subset $K$ of $M$ there is no past directed and past inextendible nonspacelike curve $x(t)$ which is totally past imprisoned in $K$.

3. Each open subset $E$ of $M$ with compact closure $\bar{E}$ is stably causal.

Proof. Theorem 2 states that 1 implies 3. Furthermore, the proof of Theorem 2 shows that 2 implies 3 .

Assume that condition 3 is valid. Let $K$ be a compact subset of $M$. There is always an open subset $E$ with $\bar{E}$ compact and $K \subset E$. Since $E$ is stably causal it is strongly causal. Therefore, there can be no future (past) directed and future (past) inextendible nonspacelike curve $x(t)$ of $E$ which is totally future (past) imprisoned in $K$. The same statement must be true for $E$ replaced by $M$. Consequently, 1 and 2 are valid and the Theorem is established.

For the next two results let $\mu$ be a Borel measure on $M$ such that $\mu(M)<\infty$. The measure $\mu$ need not be related to the Lorentz volume element on $M$. A measure $\mu$ with $\mu(M)<\infty$ always exists, compare [1].

Theorem 5. Let $(M, g)$ be a causal spacetime which satisfies condition $N$. If $\mu$ is a measure with $\mu(M)<\infty$, then for each $\varepsilon>0$ there is a closed subset $F$ of $M$ with $\mu(F)<\varepsilon$ and $M-F$ stably causal. 
Proof. A simple argument shows that without loss of generality we may assume $M$ is connected. It is known [5] that a connected manifold may be given a complete positive definite metric $\bar{g}$. The Hopf-Rinow Theorem [9] implies that if $d$ is the distance function obtained from $\bar{g}$, then all subsets of $M$ which are bounded with respect to $d$ have compact closure. Fix a point $q \in M$ and define $H_{k}=\{p \mid k-$ $1 \leqq d(q, p)<k\}$ for $k=1,2,3, \ldots$. The sets $H_{k}$ are disjoint Borel sets with compact closure. The countable additivity of $\mu$ implies

$$
\mu(M)=\Sigma \mu\left(H_{k}\right) .
$$

Consequently, there is some $m$ such that the union of all $H_{k}$ with $k \geqq m$ has measure less than $\varepsilon$. Let $F$ be the union of all $H_{k}$ with $k \geqq m$. The set $F$ is a closed set. Furthermore, $M-F$ has compact closure. The final result now follows from Theorem 2.

Corollary 6. If $(M, g)$ is strongly causal and $\mu$ is a measure with $\mu(M)<\infty$, then $M$ less a suitable set of arbitrarily small measure is stably causal.

\section{Conformal Changes}

In this section we will obtain the main result. A causal spacetime satisfying condition $\mathrm{N}$ will be shown to be conformally equivalent to a spacetime which has all nonspacelike geodesics complete.

A geodesic $x(t)$ of $M$ with $t$ an affine parameter is complete if it can be extended to arbitrary positive and negative values of its affine parameter, see [10]. A spacetime is null and timelike complete if all null and timelike geodesics are complete. When all geodesics are complete the spacetime is called geodesically complete.

The causality of $(M, g)$ and $\left(M, \Omega^{2} g\right)$ is always the same, however, the geodesics of $g$ and $\Omega^{2} g$ can be quite different.

If $x^{1}, x^{2}, \ldots, x^{n}$ are coordinates for a neighborhood $U(p)$ and $r \in U(p)$, then the $i^{\text {th }}$ coordinate of $r$ will be denoted by $x^{i}(r)$.

Lemma 7. Let $(M, g)$ be stably causal and let $f$ be a cosmic time function with $\operatorname{grad} f$ always future pointing. If $p \in M$, then there is a neighborhood $U(p)$ with coordinates $x^{1}, x^{2}, \ldots, x^{n}$ such that $x^{1}(r)=f(r)$ for all $r \in U(p)$.

In the above Lemma let $U(p)$ be the coordinate neighborhood where $x^{1}, \ldots, x^{n}$ are the local coordinates. There exists $b_{2}>b_{1}>f(p)$ and a neighborhood $V(p) \subset U(p)$ such that any future directed nonspacelike curve which intersects $\bar{V}(p)$ must also intersect $\left\{r \in U(p) \mid x^{1}(r)=c\right\}$ for all $b_{1} \leqq c \leqq b_{2}$. Assume without loss of generality that $b_{3}$ is a positive number such that any future directed nonspacelike curve $x(t)$ which intersects $\bar{V}(p)$ must have $\left|x^{i}(t)\right| \leqq b_{3}$ for all $i \geqq 2$ and all $t$ with $f(p) \leqq x^{1}(t) \leqq b_{2}$. Assume also that the coordinate domain of $U(p)$ includes all $x$ such that $f(p) \leqq x^{1} \leqq b_{2}$ and $\left|x^{i}\right| \leqq 2 b_{3}$.

Let $(x, v)=\left(x^{1}, \ldots, x^{n}, v^{1}, \ldots, v^{n}\right)$ be the natural coordinates on $T(U(p))$, the tangent bundle over $U(p)$. Let $L, \delta_{1}, \delta_{2}$, and $\delta_{3}$ be four numbers with $b_{1}<\delta_{1}<$ $\delta_{2}<\delta_{3}<b_{2}$ and $L \geqq 1$. Let $S\left(\delta_{1}, L\right)$ be the compact set of tangent vectors $(x, v)$ such that $x^{1}=\delta_{1},\left|x^{i}\right| \leqq b_{3}$ for $i \geqq 2, L=\Sigma\left(v^{j}\right)^{2}$ and $(x, v)$ is future pointing. By choosing $\delta_{1}, \delta_{2}, \delta_{3}$ sufficiently close together we may assume each future directed nonspacelike curve which intersects $\left\{x\left|x^{1}=\delta_{1},\right| x^{i} \mid \leqq b_{3}\right.$ for $\left.i \geqq 2\right\}$ also intersects 
$\left\{x\left|x^{1}=\delta_{3},\right| x^{i} \mid \leqq 2 b_{3}\right.$ for $\left.i \geqq 2\right\}$. Given any $k>0$ it is always possible to find a $C^{\infty}$ function $\Omega$ which is identically one for $r \in M$ unless $r \in U(p)$ and $\delta_{2}<x^{1}(r)<\delta_{3}$ such that any geodesic $x(t)$ of $\Omega^{2} g$ with $(x(0), \dot{x}(0)) \in S\left(\delta_{1}, L\right)$ increases its affine parameter by at least $k$ in going from the hypersurface $f(r)=\delta_{2}$ to the hypersurface $f(r)=\delta_{3}$. The metrics $g$ and $\Omega^{2} g$ are exactly the same except for $r \in U(p)$ with $\delta_{2}<x^{1}(r)<\delta_{3}$.

In the Theorem which follows we will make use of the neighborhoods $U(p)$, $V(p)$ and the numbers $b_{1}=b_{1}(p), b_{2}=b_{2}(p)$, and $b_{3}=b_{3}(p)$ which are constructed as in the above paragraphs. The numerical restrictions on $\delta_{1}=\delta_{1}(p), \delta_{2}=\delta_{2}(p)$, $\delta_{3}=\delta_{3}(p), L$, and $k$ are $b_{1}(p)<\delta_{1}(p)<\delta_{2}(p)<\delta_{3}(p)<b_{2}(p), L \geqq 1$ and $k>0$.

Let $U\left(p_{0}\right)$ and $U\left(p_{1}\right)$ be given with coordinates $x_{0}^{i}$ and $x_{1}^{i}$, respectively. If $r \in U\left(p_{0}\right) \cap U\left(p_{1}\right)$, then $x_{0}^{1}(r)=x_{1}^{1}(r)=f(r)$. However, it may hapepn that $x_{0}^{i}(r) \neq$ $x_{1}^{i}(r)$ for $i \geqq 2$. Notice that if $b_{1}\left(p_{0}\right) \leqq b_{1}\left(p_{1}\right)$ we may choose the $\delta_{i}\left(p_{0}\right)$ and $\delta_{i}\left(p_{1}\right)$ such that $\delta_{3}\left(p_{0}\right)<\delta_{1}\left(p_{1}\right)$. The corresponding conformal factors $\Omega_{0}$ and $\Omega_{1}$ will then be different from unity on disjoint sets.

Theorem 8. Let $(M, g)$ be a causal spacetime which satisfies condition $N$. There is some conformal factor $\Omega$ such that $\left(M, \Omega^{2} g\right)$ is null and timelike complete.

Proof. Assume without loss of generality that $M$ is connected. Choose a fixed $q \in M$ and let $\bar{g}$ be a complete positive definite metric on $M$ with corresponding distance function $d$ as in the proof of Theorem 5. Let $W(k)=\{r \mid k-1<d(q, r)<k\}$ and $B(k)=\{r \mid 0 \leqq d(q, r)<k\}$. The sets $W(k)$ and $B(k)$ are open subsets having compact closure. Theorem 2 implies $B(5 k)$ is stably causal and hence has a cosmic time function $f_{k}$. Assume grad $f$ is always future pointing on $B(5 k)$. The idea is to define inductively a sequence of functions $\Omega_{k}$ such that $\Omega_{k}$ is identically one except possibly on the set $\bar{W}(4 k-1) \cup \bar{W}(4 k) \cup \bar{W}(4 k+1)$. The final conformal factor $\Omega$ will be the infinite product $\Pi \Omega_{k}$ of the functions $\Omega_{k}$. Actually, for each fixed $k$ the function $\Omega_{k}$ will be defined by a finite induction and will, in fact, be the finite product of functions $\Omega_{k}^{i}$.

The definition of $\Omega_{k}$ will vary according to if $k$ is even or odd. Let $\Omega_{0} \equiv 1$.

Case 1. Let $k$ be odd

Let $g_{k}=\Omega_{0}^{2} \ldots \Omega_{k-1}^{2} g$. For each $p \in \bar{W}(4 k)$ use the cosmic time function $f_{k}$ and define $U(p), V(p), b_{1}(p), b_{2}(p)$, and $b_{3}(p)$ as described above with $U(p) \subset \bar{W}(4 k-1) \cup$ $\bar{W}(4 k) \cup \bar{W}(4 k+1)$. The sets $V(p)$ form an open cover of the compact subset $\bar{W}(4 k)$. Let $V\left(p_{1}\right), \ldots, V\left(p_{m}\right)$ be a finite subcover such that $f_{k}\left(p_{i}\right)<f_{k}\left(p_{i+1}\right)$ and $b_{1}\left(p_{1}\right) \leqq b_{1}\left(p_{2}\right) \leqq \ldots \leqq b_{1}\left(p_{m}\right)$. Choose the $\delta$ 's as above with the further restriction that $\delta_{3}\left(p_{i}\right)<\delta_{1}\left(p_{i+1}\right)$ for all $i=1, \ldots, m-1$. For each $i$ we wish to construct a function $\Omega_{k}^{i}$ which is identically one except for $r \in U\left(p_{i}\right)$ with $\delta_{2}\left(p_{i}\right)<f_{k}(r)<\delta_{3}\left(p_{i}\right)$.

Assume $\Omega_{k}^{1}, \ldots, \Omega_{k}^{i-1}$ have been defined. Let $\hat{g}=\left(\Omega_{k}^{1} \ldots \Omega_{k}^{i-1}\right)^{2} g_{k}$. Consider $x(t)$ a future directed nonspacelike geodesic of $(B(5 k), \hat{g})$ which is future and past inextendible in $B(5 k)$ and satisfies $(x(0), \dot{x}(0)) \in S\left(\delta_{1}\left(p_{i}\right), 1\right)$. Because $B(5 k)$ is stably causal there must exist some $t_{1}<0$ such that $x\left(t_{1}\right) \in W_{5 k}$. There is some positive constant $c \leqq 1$ such that if $t_{1} \leqq t \leqq 0$, then $\bar{g}(\dot{x}(t), \dot{x}(t))>c$. Therefore, there is some neighborhood $Q$ of $(x(0), \dot{x}(0))$ in $S\left(\delta_{1}\left(p_{i}\right), 1\right)$ such that if $y(t)$ is any future directed nonspacelike geodesic of $(B(5 k), \hat{g})$ which is future and past inextendible in $B(5 k)$ and $(y(0), \dot{y}(0)) \in Q \subset S\left(\delta_{1}\left(p_{i}\right), 1\right)$, then $y\left(t_{1}\right) \in W_{5 k}$ and $\bar{g}(\dot{y}(t), \dot{y}(t))>c / 2$ for all $t_{1} \leqq t \leqq 0$. 
The compactness of $S\left(\delta_{1}\left(p_{i}\right), 1\right)$ implies there is some $L \geqq 1$ such that if $x(t)$ is any future directed geodesic of $(B(5 k), \hat{g})$ with $(x(0), \dot{x}(0)) \in S\left(\delta_{1}\left(p_{i}\right), L\right)$, then $t<0$ with $x(t) \in B(k)$ and $x(s) \notin W_{5 k}$ for all $t \leqq s \leqq 0$, implies $\bar{g}(\dot{x}(t), \dot{x}(t)) \geqq 1$. The function $\Omega_{k}^{i}$ will be identically one except possibly for $r \in U\left(p_{i}\right)$ with $\delta_{2}\left(p_{i}\right)<x^{1}(r)<\delta_{3}\left(p_{i}\right)$. We require that if $x(t)$ is a geodesic of the new metric $\Omega_{k}^{i} \hat{g}$ with $(x(0), \dot{x}(0)) \in S\left(\delta_{1}\left(p_{i}\right), L\right)$, then $x(t)$ must increase its affine parameter by at least $k$ in going from the hypersurface $f(r)=\delta_{2}\left(p_{i}\right)$ to the hypersurface $f(r)=\delta_{3}\left(p_{i}\right)$. If $(x(0), \dot{x}(0)) \in S\left(\delta_{1}\left(p_{i}\right), L^{\prime}\right)$ and $L^{\prime} \leqq L$, then the geodesic $x(t)$ increases in affine parameter by at least $\left(L^{\prime}\right)^{-1} L k$ between the hypersurfaces.

Let $\Omega_{k}=\Omega_{k}^{1} \ldots \Omega_{k}^{m}$ and $g_{k+1}=\left(\Omega_{0} \Omega_{1} \ldots \Omega_{k}\right)^{2} g$. Let $x(t)$ be a future directed nonspacelike geodesic of $\left(M, g_{k+1}\right)$ which is future inextendible and has $x\left(t_{1}\right) \in B(k)$ with $\bar{g}\left(\dot{x}\left(t_{1}\right), \dot{x}\left(t_{1}\right)\right)=1$. If $t_{2}$ is the first $t$ greater than $t_{1}$ with $d(q, x(t))=4 k$, then $t_{2}-t_{1}>k$.

\section{Case 2. $k$ even}

When $k$ is even the construction of $\Omega_{k}$ is similar to the above. The difference is that instead of increasing the affine parameter of future directed geodesics we increase the affine parameter of past directed geodesics. Construct $\Omega_{k}$ such that if $x(t)$ is a past directed nonspacelike geodesic of $\left(M,\left(\Omega_{0} \ldots \Omega_{k}\right)^{2} g\right)$ having $x\left(t_{1}\right) \in B(k)$ and $\bar{g}\left(\dot{x}\left(t_{1}\right), \dot{x}\left(t_{1}\right)\right)=1$, then $x(t)$ increases its affine parameter by at least $k$ in going from $x\left(t_{1}\right)$ to the first point $x\left(t_{2}\right)$ with $d\left(q, x\left(t_{2}\right)=4 k\right.$.

Define $\Omega=\Pi \Omega_{k}$ and let $x(t)$ be a future directed nonspacelike and future inextendible geodesic of $\left(M, \Omega^{2} g\right)$. There is some $k_{0}$ such that $x(0) \in B\left(k_{0}\right)$. We wish to show $x(t)$ is defined for all $t>0$. Assume without loss of generality that $\bar{g}(\dot{x}(0)$, $\dot{x}(0))=1$. For each odd $k \geqq k_{0}$ we have $x(0) \in B(k)$. Hence $x(t)$ increases its affine parameter by at least $k$ in going from $x(0)$ to the first $t>0$ with $d(q, x(t))=4 k$. Thus, $x(t)$ is future complete. A similar argument shows $x(t)$ is past complete and finishes the proof.

\section{Geodesic Completeness}

For a restricted class of spacetime the methods used in Section 4 can be modified so that $\left(M, \Omega^{2} g\right)$ becomes geodesically complete rather than only null and timelike complete. The proofs will be omitted in this section.

Let $(M, g)$ be a two dimensional spacetime which has no closed nonspacelike curves and no closed nontimelike curves. This spacetime $(M, g)$ will be called doubly stable if there are functions $f_{1}$ and $f_{2}$ such that $\operatorname{grad} f_{1}$ is always timelike and $\operatorname{grad} f_{2}$ is always spacelike. Clearly, any open subset of the two dimensional Minkowskian space is doubly stable.

Theorem 9. Let $(M, g)$ be a two dimensional spacetime which is doubly stable. There is a conformal factor $\Omega$ such that $\left(M, \Omega^{2} g\right)$ is geodesically complete.

Corollary 10. Let $(M, g)$ be the two dimensional Minkowskian space and let $F$ be a closed subset of $M$. There is a conformal factor $\Omega$ such that $\left(M-F, \Omega^{2} g\right)$ is geodesically complete. 


\section{References}

1. Hawking, S.W., Ellis, G.F.R.: The large scale structure of spacetime. Cambridge: Cambridge University Press 1973

2. De Witt, C., De Witt, B.: Black holes. New York: Gordon and Breach 1973

3. Weinberg, S.: Gravitation and cosmology. New York: Wiley 1972

4. Sachs, R.K.: General relativity and cosmology. New York: Academic Press 1971

5. Nomizu, K., Ozeki, H.: Proc. Amer. Math. Soc. 12, 889 (1961)

6. Seifert, H.J.: Thesis, Hamburg 1968

7. Seifert, H. J.: Gen. Rel. Grav. 1, 247 (1971)

8. Clarke, C. J.S. : Proc. Camb. Phil. Soc. 69, 319 (1971)

9. Hicks, N. J.: Notes on differential geometry. New Jersey: Van Nostrand-Reinhold 1965

10. Wolf, J.A.: Spaces of constant curvature. New York: McGraw-Hill 1967

Communicated by J. Ehlers

Received August 18, 1975 